\title{
No Effect of Cycling Shoe Outsole Stiffness On Sub- and Supra-Maximal Cycling Performance Parameters.
}

\section{Manon Varvat}

Universite Savoie Mont-Blanc

Pierre Samozino

Universite Savoie Mont-Blanc

Frédérique Hintzy ( $\nabla$ frederique.hintzy@univ-smb.fr)

Université Savoie Mont-Blanc https://orcid.org/0000-0001-8080-4217

\section{Research Article}

Keywords: Efficiency, sprints, comfort, shoe-pedal interface, non-cyclists

Posted Date: December 15th, 2021

DOl: https://doi.org/10.21203/rs.3.rs-1145206/v1

License: (c) (i) This work is licensed under a Creative Commons Attribution 4.0 International License.

Read Full License 
TITLE:

4 No effect of cycling shoe outsole stiffness on sub- and supra-maximal cycling performance 5 parameters.

6

7 BRIEF RUNNING HEAD:

8 Cycling shoe outsole stiffness and performance.

9

10 AUTHORS and INSTITUTIONS:

11 Varvat M, Samozino P, Hintzy F

12

13 Manon VARVAT: Inter-University Laboratory of human Movement Biology, University

14 Savoie Mont-Blanc, F-73376 Le Bourget-du-Lac, France

15 varvatmanon@gmail.com

16

17 Frédérique HINTZY: Inter-University Laboratory of human Movement Biology, University 18 Savoie Mont-Blanc, F-73376 Le Bourget-du-Lac, France

$19+334797581$ 34; frederique.hintzy@univ-smb.fr

20 ORCiDs : 0000-0001-8080-4217

21 LinkedIn : https://www.linkedin.com/in/frédérique-hintzy-865b5727/

22 Twitter; @FHintzy

24 Pierre SAMOZINO: Inter-University Laboratory of human Movement Biology, University 25 Savoie Mont-Blanc, F-73376 Le Bourget-du-Lac, France 
+3347975 8177; pierre.samozino@univ-smb.fr

ORCiDs : 0000-0002-1665-870X

LinkedIn : https://www.linkedin.com/in/pierre-samozino-73485142/

Twitter : https://twitter.com/PierreSamozino

\section{CORRESPONDING AUTHOR:}

\section{$\underline{\text { Frédérique HINTZY }}$}

Inter-University Laboratory of human Movement Biology, University Savoie Mont-Blanc, F73376 Le Bourget-du'Lac, France

+33479758134; frederique.hintzy@univ-smb.fr

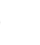

TITLE: No effect of cycling shoe outsole stiffness on sub- and supra-maximal cycling 1 performance parameters.

\section{ABSTRACT}

4 Background: The aim of this study was to test the effects of cycling shoe outsole stiffness on both performance and comfort parameters during sub- and supra-maximal cycling tests. 6 Methods: Two groups of recreational women tested three cycling shoe conditions with 47 differing outsole stiffness. One group of 8 women performed four cycling tests of 3 min 48 composed of two intensities (100 and $140 \mathrm{~W})$ and two pedaling rates (70 and $100 \mathrm{rpm})$ for each 49 pair of shoes. Metabolic and subjective perception of comfort measurement was evaluated with 0 each shoe. Another group of 12 women performed 6-s all-out sprints against two external 
resistances $(0.4$ and $0.7 \mathrm{~N} / \mathrm{kg})$ to determine force-velocity relationships with the three cycling shoe conditions. Results: The main findings are that the stiffness of the investigated outsole cycling shoes (i) does not influence cycling performance whatever the test (ii) while the perception of comfort is largely degraded compared to the most flexible shoe. Conclusion: Maximizing stiffness should no longer be of the highest design principal for beginners or recreational women cyclists.

KEYWORDS: Efficiency; sprints; comfort; shoe-pedal interface; non-cyclists.

\section{BACKGROUND}

In cycling, the majority of the energy imparted to the bike occurs through the link between the foot and the crank through the shoe-pedal interface [1-2]. This interface allows the transmission of the force developed by the cyclist's lower limbs to the crank, and then to the chainring. The resultant force applied to the crank can be divided into an effective force perpendicular to the crank and directed in the direction of rotation, and ineffective forces parallel to the crank. Consequently, the shoe-pedal system influences both muscular force production and mechanical force effectiveness (ratio of effective force/resultant force), making it an essential interface. Improving the transmission of a force through an interface is possible by minimizing the loss of energy in the deformation of this interface. From a mechanical point of view, stiffness is the ability of a solid object to resist deformation when it is subjected to mechanical stresses. It mainly depends on the geometry and the elasticity modules of the material used. Contextualized to cycling, all the subcomponents constituting the shoe-pedal interface should thus be stiff, with the single purpose of transmitting force: the sole of the shoe as a support between the foot and the pedal, and the linkage of the shoe with the pedal [1-3]. 
The present study focuses only on the outsole of the shoe, i.e., the structural portion of the shoe that transfers the force.

There are many claims by cyclists and industry professionals about the advantages of outsole stiffness using composite materials (such as carbon fiber) that ensure stiffness while remaining light and thin. The study of Jarboe and Quesada [4] compared the influence of the stiffness of cycling shoe midsoles on plantar pressure during an exercise of $400 \mathrm{~W}$ at $90 \mathrm{rpm}$. Firstly, dynamic mechanical testing indicated that cycling shoes with carbon fiber composite midsoles were significantly stiffer than cycling shoes made with a more traditional plastic sole, in both longitudinal bending and three-point bending. They then showed that these stiffer cycling shoes induced peak plantar pressures $18 \%$ higher in the forefoot region (first metatarsal head and hallux) than did the other shoe with a plastic midsole. The limited ability to flex the metatarsophalangeal joint under load with a stiff sole, and thereby conform to foot contours so that loads may not be spread over larger areas, may explain this higher plantar pressure [4-5]. The plantar pressure increase is consistent with an optimization of pedaling kinetics, since this forefoot region was identified as the major force-contributing structure of the foot [5], and the relative contribution of this region increases with an increase of power output from 100 to 400 W [6].

The scientific literature showed that the increase in the longitudinal bending stiffness of sole athletic shoes results in an improvement in performance during maximal intensity shortterm pushing movements, such as sprinting and jumping [7-10]. The causal mechanism is the reduction in the range of motion of the metatarsophalangeal joints, which reduces the amount of energy dissipation at this joint. One might hypothesize that stiffer-outsole cycling shoes also transfer power more efficiently to the crank, leading to better performance. It is difficult to answer this question correctly based strictly on the literature. On the one hand, the studies that tested large differences in shoe stiffness had to use running shoes for the minimum stiffness 
condition [e.g. 6, 11-16]. But the running shoe inevitably requires the use of flat or toe-clip

102 pedals, while the cycling shoes used to qualify stiffness require clipless pedals. Furthermore,

103 the differences between running and cycling shoes were not only in terms of the stiffness of the

104 outsole but also the thickness and width of foam between in- and outsole. Consequently, these

105 studies cross the stiffness effect with the type of shoe-pedal linkage and/or other shoe

106 characteristics. The results are thus highly variable depending on the equipment tested. On the

107 other hand, some studies only use road cycling shoes with clipless pedals, investigating a

108 spectrum of commercially available shoes with different longitudinal bending stiffness [17] or

109 comparing standard insoles vs. carbon fiber insoles or a specific orthosis placed in the same

110 road cycling shoe [5-18-20]. These two procedures have the advantage of only testing the

111 stiffness effect, but the disadvantage of doing so in a low stiffness range, i.e., with very low

112 stiffness differences between the shoe conditions. Indeed, all the commercially available road

113 cycling shoes can already be characterized as stiff compared to other type of sports shoe, and

114 only stiff to very stiff conditions are therefore compared when a full carbon fiber insole is added.

115 Additional data presented in Hurt's thesis [21] confirm this limit: most of the subjects testing

116 the spectrum of commercially available road shoes reported that all of the shoes felt similar,

117 comfortable, and stiff enough for cycling sprinting, and they were not able to correctly guess

118 the relative stiffness of each shoe. These studies reach a consensus: little or no effect of sole

119 stiffness on parameters of cycling performance. Hurt and Kram [17] and Koch et al. [20]

120 showed that increased longitudinal bending stiffness of cycling shoe soles had no effect on

121 sprint performance during short uphill sprints and 20-s Wingate anaerobic power test,

122 respectively. Moreover, Anderson and Sockler [18] found that, at a given mechanical power

123 output, there were no statistically significant differences in oxygen consumption, expired

124 ventilatory volume, or heart rate between the conditions with/without foot stiffness orthoses.

125 Fletcher et al. [19] complemented this analysis by testing the torsional stiffness of the road 
shoes during sub-maximal cycling. They did not find any statistically significant differences between either the knee moment or the gross efficiency in the two stiffness conditions. To conclude, the cycling outsole shoe stiffness can affect pedaling kinetics [4] without affecting performance parameters in both supra- and sub-maximal conditions [17-20]. consequences to high stiffness. Firstly, the repetitively occurring high pressure under the forefoot attained with the stiffer outsole may be the cause of localized ischemia, paresthesia,

133 and metatarsalgia syndromes of the forefoot frequently observed in cyclists [1, 3-5, 22-28].

134 Secondly, if a stiff sole transfers forces more efficiently, it also transfers more vibrations.

135 Vibrations can also cause musculoskeletal disorders [29]. Ultimately, both high plantar pressure 136 and vibration transmission also affect comfort [3, 30-31]. A shoe that flexes more to conform 137 to foot contours, and that dampens vibration over rough terrain, is theorized to be a more 138 comfortable and "safer" shoe.

139 To optimize both cycling performance and shoe comfort, investigations are needed to 140 clarify which optimal value of outsole stiffness is required. Thus, it would be necessary to test 141 a large range of outsole cycling shoe stiffnesses in different power output conditions. Moreover, 142 women are too little studied in cycling literature, and the results of studies obtained on men 143 cannot be completely extrapolated to women, particularly with regard to mechanical data of 144 mass, stiffness, and power. For example, the maximum power developed during sprinting is $145500-800 \mathrm{~W}$ in high-level women cyclists [32] vs. 1100-1400 W in men [33-34]. Consequently, 146 women cyclists may exert less pressure on the outsole, which would deform it to a lesser degree, 147 compared to male cyclists. Sole stiffness is probably even less important in women, since they 148 are generally less powerful. It is relevant to focus on the influence of shoe stiffness in woman 149 cyclists on both performance and comfort by testing a wide spectrum of outsole stiffnesses 150 greater than those offered by commercially available cycling shoes. 
The aim of this study was thus to test the effects of cycling shoe outsole stiffness on both

152 performance and comfort during sub- and supra-maximal cycling tests in women. The 153 performance was quantified by the external force production capacities (Force-Velocity-Power 154 relationship) for the supra-maximal test, and by energetical and perceptual parameters for the 155 sub-maximal test. It was expected that the shoe stiffness should be adapted to the power output: 156 no effect during the sub-maximal test since the power output is very low, but a possible decrease 157 of some mechanical parameter developed during the sprint with the more flexible outsole. A second hypothesis was that the too stiff outsole lead to perceived discomfort.

\section{MATERIAL AND METHODS}

Three cycling shoe conditions were tested with highly different outsole stiffnesses: very of outsole materials and their density and was quantified by material testing techniques using certified procedures (resistance at rupture, modulus of elasticity, and elongation at rupture index; Table 1). This dynamic shoe testing showed that the $\mathrm{S}+$ shoe was stiffer than the $\mathrm{S}$ and

167 S- shoes irrespective of the test, and that, inversely, the S- shoe was very flexible. To illustrate this difference in stiffness in practical terms, the $\mathrm{S}$ - shoe can be deformed so as to fold the shoe back on itself (toe-heel contact) with the force of a single hand.

Table 1: Mean mechanical characteristics of the shoes tested.

\begin{tabular}{|c|c|c|c|}
\cline { 2 - 4 } \multicolumn{1}{c|}{} & S- & $\mathrm{S}$ & $\mathrm{S}+$ \\
\hline Outsole material & Polyamide & Thermoplastic $+5 \%$ & Full carbon \\
& & fiberglass & \\
\hline Density $\mathrm{g} / \mathrm{cm}^{3}$ & 1.14 & 1.23 & 1.78 \\
\hline
\end{tabular}




\begin{tabular}{|c|c|c|c|}
\hline $\begin{array}{l}\text { Resistance at rupture by traction } \\
\qquad\left(\text { da N/mm }{ }^{2}\right)\end{array}$ & 6 & 14.5 & 415 \\
\hline $\begin{array}{l}\text { Resistance at rupture by flexion } \\
\qquad\left(\text { da N/mm }{ }^{2}\right)\end{array}$ & 5.5 & 13.8 & - \\
\hline $\begin{array}{l}\text { Modulus of elasticity by traction } \\
\qquad(\text { da N/mm })\end{array}$ & 180 & 4960 & 23100 \\
\hline $\begin{array}{l}\text { Modulus of elasticity by } \\
\text { flexion }\left(\text { da } \mathrm{N} / \mathrm{mm}^{2}\right)\end{array}$ & 140 & 276 & - \\
\hline $\begin{array}{l}\text { Elongation at rupture by traction } \\
\qquad(\%)\end{array}$ & 200 & $>10$ & 1.8 \\
\hline
\end{tabular}

172 Legend: Shoe stiffness measurements were collected under controlled conditions and in two

173 different configurations using dynamic hydraulic tensile testing machines.

175 The three pairs of cycling shoes were manufactured by Mavic SAS (Annecy, France) in the

176 same size (European size $38^{1 / 3}$ ), modifying only the material used in the outsole of a shoe model

177 available on the market (Ksyrium Elite W). Consequently, the three shoes were identical except

178 for the outsole material and, thus, longitudinal bending stiffnesses. However, it should be noted

179 that the color of the outsole differed: $\mathrm{S}+$ : black; S and S-: white. These three outsole stiffness

180 conditions were tested in both sub- and supra-maximal cycling situations during two

181 independent studies (two protocols and two different populations). The participants were

182 blinded to the outsole characteristics.

184 Sub-maximal exercise:

185 Participants: 
186 Eight women (age $21.3 \pm 1.8$ years, height $164.4 \pm 5.4 \mathrm{~cm}$, weight $57.5 \pm 5 \mathrm{~kg}$ ) participated in

187 this study. They were physically active in different endurance sport activities, but none was

188 specifically trained in cycling. However, they can be qualified as recreational cyclists because

189 all have all ridden on various occasions. All participants were free of musculoskeletal pain or

190 injury during the study. In addition, all provided written informed consent, and the local ethics

191 committee approved both sub and supra-maximal protocols.

192 Experimental protocol:

193 The sub-maximal tests were carried out during a single laboratory session. Each participant

194 warmed-up and familiarized themselves with the cycle ergometer for $10 \mathrm{~min}$ at self-selected

195 sub-maximal intensity with the S shoe. After 5 min of passive rest, they performed, for each

196 pair of shoes, four cycling tests of 3 min composed of two intensities (100 and $140 \mathrm{~W})$ and two

197 pedaling rates ( 70 and $100 \mathrm{rpm}$ ), i.e., 12 conditions for a total duration of $36 \mathrm{~min}$. The order of

198 shoe conditions was randomized and counterbalanced, and 5 min of active rest was imposed

199 between the shoe conditions. For a shoe condition, the order of intensity and pedaling rate was

200 also randomized and counterbalanced, without a rest period between conditions. Participants

201 remained seated with both hands on the tops of the cycle ergometer handlebars, and they were

202 obliged to maintain the imposed pedaling rate given through visual feedback.

\section{Data collection and data analysis:}

204 A standard friction loaded cycle ergometer (Monark type 818 E, Stockholm, Sweden) with clip205 in pedals (ZXellium, Mavic, France) and circular chainring (Shimano XTR, France, 44 teeth) 206 was used.

207 Metabolic measurement was performed with a portable breath-by-breath device (Cosmed K $4 \mathrm{~b}^{2}$, 208 Rome, Italy) throughout the entire test. Two-point calibration of the metabolic measurement 209 system was performed prior to each session using room air and a gas mixture of known 210 composition $\left(5 \% \mathrm{CO}_{2}, 16 \% \mathrm{O}_{2}\right)$. Expired volume was calibrated using a 3-L syringe. Oxygen 
211 consumption $\left(\dot{\mathrm{VO}}_{2}\right)$ was averaged during the last $60 \mathrm{~s}$ of each condition of $3 \mathrm{~min}$ at a steady

212 state and expressed in $\mathrm{ml} \cdot \mathrm{min}^{-1} \cdot \mathrm{kg}^{-1}$.

213 The perception of comfort felt with each shoe was evaluated after use of the shoe during the

214 rest period. A visual analogue scale (VAS, $10 \mathrm{~cm}$ ) from 0 (very uncomfortable or no discomfort)

215 to 10 (very comfortable or unbearable pain) was used [35].

\section{Statistical analysis:}

217 All data are presented as mean \pm standard deviation (SD). Distribution normality and sphericity 218 were tested with Shapiro-Wilk's and Mauchly's test, respectively. When sphericity was not 219 respected, the Greenhouse-Geisser correction was applied. Comfort data did not follow normality, a nonparametric Friedman test was then performed on each cycling condition to test 221 the shoe effect. The $\dot{\mathrm{V}} \mathrm{O}_{2}$ data followed normality and respected sphericity. Then, two-factor 222 repeated-measures ANOVA (shoe and intensity) for each pedaling rate (70 and $100 \mathrm{rpm}$ ) was 223 performed, and Holm's post hoc test was used to highlight significant differences between 224 conditions. The magnitude of the observed differences across shoes was quantified using effect 225 size statistics (ESs, Cohen's $d$ ), with the thresholds for small, moderate, and large effects being 226 set at $0.2,0.5$, and 0.8 , respectively, and with $95 \%$ confidence intervals [CI] around the effect 227 sizes [36]. For all statistical analyses, an alpha value of 0.05 was accepted as the level of 228 significance. The data were processed in Excel and the statistical analyses in JASP.

\section{Supra-maximal study:}

\section{Participants:}

232 A total of 12 women (age $20 \pm 1.8$ years, height $165.5 \pm 4.1 \mathrm{~cm}$, weight $60.9 \pm 6.4 \mathrm{~kg}$ ) participated in the study. All specificities and information are similar to the sub-maximal study.

\section{Experimental protocol:}


The supra-maximal tests were carried out during a single laboratory session. Each participant

236 performed a 15-min warm-up protocol with the S shoe (self-selected sub-maximal intensity for

$23710 \mathrm{~min}$, then acceleration of $15 \mathrm{~s}$ ) followed by $5 \mathrm{~min}$ of passive rest before the start of the force-

238 velocity test. This test consisted of six all-out sprints of $6 \mathrm{~s}$ each, interspersed with 4-min rest

239 periods. Each sprint was differentiated by the shoe condition ( + +, S and $\left.\mathrm{S}_{-}\right)$and by the external

240 resistances applied to the flywheel $(0.4 \mathrm{~N} / \mathrm{kg}$ and $0.7 \mathrm{~N} / \mathrm{kg}$ of body weight $)$. The six sprints were

241 performed in randomized and counterbalanced order. The start position was standardized with

242 the crank of the right pedal still at $45^{\circ}$ after the top dead center. At a signal given by the

243 experimenter, participants were asked to pedal as fast as possible, that is, to produce the highest

244 explosive force on the pedal, until told to stop. They were also instructed to stay seated on the

245 saddle during the whole test. The experimenters vigorously encouraged the participants

246 throughout the duration of each sprint.

\section{Data collection and data analysis:}

248 A standard friction loaded cycle ergometer (Monark type 818 E, Stockholm, Sweden) with clip249 in pedals (ZXellium, Mavic, France) and circular plate (Shimano XTR, France, 44 teeth) was 250 specifically equipped with an optical encoder (Hengstler type, RIS IP50, Aldingen, Germany)

251 for the measurement of instantaneous flywheel displacement, and a strain gauge (typical MFG 252 interface, Scottsdale, AZ, United States) for measurement of the instantaneous frictional force 253 applied by the belt to the flywheel (for more detail, see 37 ). The saddle height was adjusted to 254 individual anthropometric characteristics [38].

255 The pre-processed data averaged over each pedal downstroke (friction force $(\mathrm{N})$, friction power 256 (W), displacement (m), time (s), and velocity $\left.\left(\mathrm{m} \cdot \mathrm{s}^{-1}\right)\right)$ were sampled at $200 \mathrm{~Hz}$ and stored on a 257 PC via software Sprint bicycle and a 12-bit analog-to-digital interface card. The first pedal stoke 258 was deleted, since it was incomplete. The speed was smoothed using a 50-ms moving averaging 259 window. First order derivatives of the velocity were calculated to obtain flywheel acceleration. 
The inertia constant $(13.4 \mathrm{~N})$ was added to the acceleration to determine the inertia force. Both inertia force and mechanical resistance $(0.2 \mathrm{~N})$ were added to the friction force measured by the

262 belt to determine the total force. Only one force-velocity relationship per participant and per 263 shoe was determined by mixing the data of the two external resistances [37].

264 Six mechanical parameters were calculated per linear force-velocity relationship: F0 and V0 265 (respectively, theoretical maximum force at zero velocity and theoretical maximum velocity at 266 zero force), Pmax (theoretical maximum power in $\mathrm{W} \cdot \mathrm{kg}^{-1}$ ) corresponding to 0.25F0V0 [39], 267 Fopt (optimal force at Pmax: 0.5F0), Vopt (optimal speed at Pmax: 0.5V0), and the average 268 force obtained at the first entire pedal downstroke (F1).

\section{Statistical analysis:}

270 All data are presented as mean \pm SD. All parameters followed normality distribution (Shapiro271 Wilk test). When sphericity was not respected, the Greenhouse-Geisser correction was applied. 272 One-factor repeated-measure ANOVA was performed to detect significant shoe effects, and 273 Holm's post hoc test was used to highlight significant differences between conditions. The 274 magnitude of the observed differences across shoes was quantified using effect size statistics 275 (ESs, Cohen's $d$ ), with the thresholds for small, moderate, and large effects being set at 0.2, 0.5, 276 and 0.8 , respectively, and with 95\% confidence intervals [CI] around the effect sizes [36]. For 277 all statistical analyses, an alpha value of 0.05 was accepted as the level of significance. The data 278 were processed in Excel and the statistical analyses in JASP.

280 RESULTS

\section{Sub-maximal study:}

282 Table 2 presents VAS perceived comfort and $\dot{\mathrm{VO}}_{2}$ data for each shoe and each cycling 283 condition. Results from the nonparametric Friedman test revealed no significant outsole 284 stiffness effect on the perceived comfort, but an important tendency ( $p=0.066)$. Cohen's $d$ and 
95\%CI showed a large effect $(-1.04[-1.91 ;-0.18])$ between $\mathrm{S}-$ and $\mathrm{S}+$ outsoles and a moderate effect $(-0.61[-1.4 ; 0.14])$ between $\mathrm{S}$ - and $\mathrm{S}$ : the less stiff sole $\mathrm{S}$ - is perceived to be more comfortable than the stiffer soles $\mathrm{S}+$ and $\mathrm{S}$. On the other hand, the effect between $\mathrm{S}$ and $\mathrm{S}+$ is small. $\dot{\mathrm{V}} \mathrm{O}_{2}$ is not significantly influenced by the shoe condition ( $p$ from 0.12 to 0.72 ) or by a cross shoe-intensity effect ( $p=0.67$ ), and Cohen's $d$ are between 0.04 and 0.49 , i.e. negligible to small.

Table 2: Values of perception of comfort and $\dot{\mathrm{VO}}_{2}$ with each shoe for each cycling condition.

\begin{tabular}{|c|c|c|c|c|}
\hline & & S- & $\mathbf{S}$ & $\mathbf{S}+$ \\
\hline Confor & Scale (0-10) & $1.15 \pm 1.46$ & $2.10 \pm 1.83$ & $2.99 \pm 2.23$ \\
\hline & 70rpm/100W & $30.9 \pm 2.75$ & $31.9 \pm 1.38$ & $31.7 \pm 2.07$ \\
\hline$\dot{\mathbf{V}}$ & 70rpm/140W & $35.6 \pm 2.76$ & $35.9 \pm 2.16$ & $35.7 \pm 1.95$ \\
\hline (ml.min- & $100 \mathrm{rpm} / 100 \mathrm{~W}$ & $36.8 \pm 1.92$ & $38.0 \pm 3.19$ & $37.4 \pm 1.50$ \\
\hline$\left.{ }^{1} \cdot \mathrm{kg}^{-1}\right)$ & 100rpm/140W & $39.7 \pm 2.53$ & $40.2 \pm 3.30$ & $40.0 \pm 2.02$ \\
\hline
\end{tabular}
of the outsole. Data are means \pm SD.

Supra-maximal study:

297 The mechanical parameters obtained with each shoe are presented in Table 3. Statistical analysis did not show any significant shoe effect for all mechanical parameters ( $p$ from 0.26 to 0.86), and Cohen's $d$ are between 0.01 and 0.26, i.e. negligible to small.

Table 3: Values of mechanical parameters measured during the sprints with each shoe. 


\begin{tabular}{|c|c|c|c|}
\cline { 2 - 4 } \multicolumn{1}{c|}{} & S- & S & S+ \\
\hline V0 (rpm) & $225 \pm 17.3$ & $222 \pm 21.1$ & $221 \pm 18.1$ \\
\hline Vopt (rpm) & $112 \pm 8.67$ & $111 \pm 10.5$ & $110 \pm 9.04$ \\
\hline F0 (N) & $106 \pm 11.88$ & $107 \pm 16.2$ & $109 \pm 14.1$ \\
\hline Fopt (N) & $53.1 \pm 5.94$ & $53.7 \pm 8.08$ & $54.7 \pm 7.03$ \\
\hline Pmax (W/kg) & $9.86 \pm 1.38$ & $9.81 \pm 1.40$ & $9.97 \pm 1.47$ \\
\hline F1 (N) & $83.3 \pm 9.34$ & $83.3 \pm 10.7$ & $83.1 \pm 9.10$ \\
\hline
\end{tabular}

Legend: $\mathrm{S}-, \mathrm{S}$, and $\mathrm{S}+$ corresponded, respectively, to the lowest, moderate, and highest stiffness

303 of the outsole. Data are means $\pm \mathrm{SD}$.

304

\section{DISCUSSION}

The main finding of this study was that the stiffness of the tested outsole cycling shoes does not influence beginner or recreational women cycling performance investigated through oxygen consumption during submaximal exercise and external force capacities during supramaximal tests.

This result is not surprising regarding the comparison between the two stiffest shoes of

311 the three tested (S+ vs. S). These two shoes are marketed by a manufacture, the S shoe for

312 leisure cyclists and the $\mathrm{S}+$ for competitive cyclists. Thus, both have the same invariable characteristic of a road cycling shoe of being stiff, the $\mathrm{S}+$ shoe being stiffer than the $\mathrm{S}$ shoe. No

314 study has shown an effect for the stiffness of commercially available in- and/or outsoles in road 315 cycling shoes on performance parameters during sprinting or sub-maximal tests [17-20]. Since 316 these studies test male cyclists developing high levels of power, it is not surprising that the 317 effect of lack of stiffness is also observed in our non-cycling women developing low power 318 levels. Stiffness been the capacity of the support to deform or not under the application of a 
mechanical stress, the low power developed by the women in this study does not allow a

320 discrimination of the effect of the stiffness between the $\mathrm{S}+$ and $\mathrm{S}$ outsoles tested. Alone this

321 result is interesting, since it shows that women beginner or recreational cyclists can use road

322 cycling shoes marketed to be less rigid, while maintaining the same level of performance as

323 other more rigid shoes. However, cycling shoes with a higher bending stiffness have been

324 shown to generate more discomfort and potentially aggravate metatarsalgia or ischemic

325 syndromes due to the higher peak plantar pressure on the forefoot [1, 3-5, 22-28]. Consequently,

326 testing commercial road cycling shoes has the advantage of providing cyclists with usable

327 results on the balance between performance and foot pathology. However, this procedure also

328 presents the disadvantage of testing a restricted range of stiffness, i.e., from stiff to very stiff.

329 This limit was introduced by Hurt and Kram [17], who suggest carrying out a new study to

330 investigate the cycling shoe sole stiffness at which sprint performance begins to diminish. In

331 practical terms, it would be necessary to test shoes with mechanical characteristics of stiffness

332 between that of a running shoe and that of a nylon outsole road cycling shoe

333 The present S- shoe responds to this characteristic: retaining all the specificities of a road

334 cycling shoe (shoe-pedal linkage, shape, tightening), while reducing the stiffness of the outsole

335 as much as possible. The present prototype makes it feasible to provide the most flexible road

336 cycling shoe possible while maintaining the invariants of pedaling. It could be referred to as a

337 flexible "slipper" road cycling shoe, i.e., with a very low resistance to deformation. A major

338 result of this study was that the low longitudinal bending stiffness of the S- outsole had no

339 effect on sprint performance in present women. Our results may be counterintuitive in view of

340 the literature on the testing of flexible shoes. Burns and Kram [12] compared two extreme shoe

341 types -highly flexible running shoes and carbon-soled cycling shoes- and showed that the

342 flexible shoe decreases 100-m sprint performance in men. However, it seems problematic to

343 conclude that the stiffness of these shoe is the only factor responsible for improving 
344 performance, since both conditions proposed different pedal linkage. Moreover, the subjects were male cyclists who develop high power levels, therefore overly high mechanical stress compared to the low resistance to deformation of their flexible running shoe.

Thus, it seems that the resistance to deformation of the current $\mathrm{S}-$ flexible outsole is

348 sufficient for the low maximal power output attained by the women tested (about $600 \mathrm{~W}$ ). An

349 analysis of pedaling kinetics supports and complements this hypothesis. The resultant force

350 vector applied by the lower limb passes mostly to the metatarsophalangeal joint, directly over

351 the pedal axis, as shown by the plantar pressure studies [5-6]. The longitudinal bending stiffness

352 of the outsole over its entire length therefore has little or no influence. The only important area

353 of the outsole is thus the surface just above the pedal. The clip-in pedals form a small rigid

354 block under this joint, making it an almost non-deformable support during the pushing cycle

355 phase. Thus, even if the $\mathrm{S}-$ outsole has a low longitudinal bending stiffness, it remains thin and

356 therefore not vertically deformable. Consequently, the stiffness of the outsole in its entirety has

357 no influence on maximal cycling performance, provided that it remains non-deformable

358 precisely at the junction with the pedal.

359 These kinetic explanations also support the non-effect of outsole stiffness observed

360 during the sub-maximal tests, the powers imposed being very low at 100 and $140 \mathrm{~W}$. Since the

361 outsole was not deformed by the application of the force produced by the women for these low

362 constraints, it is logical that the oxygen consumption is significantly influenced by the outsole

363 stiffness, irrespective of power or pedaling rate sessions. Our result agrees with those of other

364 studies (i) comparing different stiffness insoles placed in road cycling shoes [18-19] and also

365 (ii) comparing running shoes with road cycling shoes [14-16]. Only the studies by Jorge and

366 Hull [40] and Cruz and Bankoff [13], to my knowledge, have shown conflicting results on

367 EMG: soft-soled shoes (running or tennis shoes) required more leg muscle activity (quadriceps, 
hamstrings) than soft-soled shoes. However, the pedal linkages are different, which is the purpose of these two studies.

The lack of effect of outsole stiffness on both supra- and sub-maximal cycling performance allows other important parameters in cycling to be considered: comfort and 372 pathology. A tendency effect of stiffness was shown on the perceived comfort and a significant 373 difference appears when comparing the two most distant outsoles in stiffness with a Holm's 374 post hoc test. The less stiff outsole $\mathrm{S}_{-}$is perceived to be more comfortable than the stiffer outsole S+, with this effect being identified as large by the Cohen's d test. The explanatory hypotheses may be better plantar pressure distribution on a large part of the sole or lower peak plantar pressure with the less stiff outsole. This notion of perceived comfort is an important parameter for training and/or performance. The effect of outsole stiffness on perceived comfort during a cycling test is evaluated for the first time by the present study. Uden et al. [30] showed from a questionnaire that an overly stiff cycling shoe creates discomfort, given that $54 \%$ of the cyclists questioned felt foot pain with their stiff shoes. In fact, it is the link between comfort and pathology that this study questioned. There is a consensus that decreasing the stiffness of cycling shoe soles may help to prevent or alleviate the ischemia, paresthesia, or metatarsalgia that can occur in cyclists [1, 3-5, 22-28]. Thus, evaluating both performance and comfort seems to be the best solution to guide cyclists in choosing the best shoe for them. For the women in 386 the present study, the best outsole maybe the less stiff, since it does not deteriorate their cycling performance and improves their perceived comfort, irrespective of the power output.

This recommendation cannot be strictly applied to male cyclists or elite women cyclists,

389 i.e., cyclists developing high power output, which would thus deform the soles to a greater 390 degree. However, it would be interesting to adapt cycling shoe stiffness to the power developed 391 by those wearing them, in order to determine an optimal ratio of performance to shoe comfort. 392 The hypothesis that different cyclists would require different shoe stiffnesses for their maximal 
393

performance can also be supported. One could also argue that the stiffness for racing shoes

394 should be different from that for training shoes, given the length of time required on the bike

395 for elite cyclists to train. Using substantially less stiff cycling shoes during training would 396 theoretically reduce the risk of foot pathologies and improve comfort. Finally, this study may 397 also help manufacturers to improve the design of road cycling shoes by no longer focusing 398 solely on this stiffness criterion.

\section{CONCLUSIONS}

To conclude, the stiffness of the outsole cycling shoes investigated does not influence current parameters of performance during sub- and supra-maximal tests in women. Similar results had been shown on cyclists during short uphill sprints [17], 20-s Wingate anaerobic power test [20] or submaximal cycling session [18-19]. This result is interesting since it shows that beginners or recreational women cyclists can use road cycling shoes marketed as being less rigid, while maintaining the same level of performance as other more rigid shoes. Cycling shoe outsoles can ultimately be optimized for comfort and injury prevention, as well as high performance. Further studies are needed to compare these results with other populations, such as competitive women cyclists who develop more power output, and other conditions, e.g., different gradients.

\section{LISTE OF ABBREVIATIONS}

S-: very low stiffness outsole; S: moderate stiffness outsole; $\mathrm{S}+$ : high stiffness outsole; $\dot{\mathrm{V}} \mathrm{O}_{2}$ :

Oxygen consumption; VAS: visual analogue scale; SD : standard deviation; ANOVA: analysis of variance ; ESs: effect size statistics ; CI: confidence intervals; F0: theoretical maximum force at zero velocity; V0: theoretical maximum velocity at zero force; Pmax : theoretical maximum 
417 power; Fopt: optimal force at Pmax; Vopt: optimal speed at Pmax; F1: average force obtained 418 at the first entire pedal downstroke.

\section{DECLARATIONS}

Ethics approval and consent to participate:

422 Ethical permission to undertake this work was granted by the University of Savoie Mont-Blanc. 423 All participants consented to take part in this research and for the findings to be published.

Consent for publication:

Consent to publish research findings was obtained from all participants prior to their participation in this project. No personal identifiable data is included within this publication.

\section{Availability of data and materials:}

All data can be made available from the corresponding author upon reasonable request.

\section{Competing interests:}

433 The authors declare no conflicts of interest.

\section{Funding:}

436 This research received no specific grant from any funding agency in the public, commercial, or not-for-profit sectors.

\section{Authors' contributions:}

440 All authors contributed to the design, registration, conduct and reporting of this research. All 441 authors read and approved the final manuscript. 
443 Author details:

444 Inter-University Laboratory of human Movement Biology, University Savoie Mont-Blanc, F44573376 Le Bourget-du-Lac, France.

446

447 Acknowledgements:

448 We thank MAVIC SAS (Annecy, France) for having provided the cycling shoes. However, 449 MAVIC SAS had no role or involvement in grant support, research design, results and 450 publication.

\section{REFERENCES}

454 1. Gregor RJ, Wheeler JB. Biomechanical factors associated with shoe/pedal interfaces. Sports 455 Med. 1994;17(2):117-31.

456

2. Ruby P, Hull ML, Kirby KA, Jenkins DW. The effect of lower-limb anatomy on knee loads 458 during seated cycling. J Biomech. 1992;25:1195-207.

460 3. Sanner WH, O’Halloran WD. The biomechanics, etiology, and treatment of cycling injuries. 461 J Am Podiatr Med Assoc. 2000;90(7):354-76.

462

463 4. Jarboe NE, Quesada PM. The effects of cycling shoe stiffness on forefoot pressure. Foot \& 464 Ankle Int. 2003;24(10):784-8. 
5. Sanderson DJ, Cavanagh PR. An investigation of the in-shoe pressure distribution during

467 cycling in conventional cycling shoes or running shoes. In: Bengt J, editor. Biomechanics X-B. 468 Human Kinetics Publishers. Champaign IL; 1987. P.903-07.

469

470 6. Hennig EM, Sanderson DJ. In-shoe pressure distributions for cycling with two types of 471 footwear at different mechanical loads. J Appl Biomech. 1995;11(1):68-80.

472

473 7. Enders H, Vienneau J, Tomaras EK, Koerger H, Nigg S, Nigg B. Soccer shoe bending 474 stiffness significantly alters game-specific physiology in a 25-minute continuous field-based 475 protocol. Footwear Sci. 2015;7(supl):S91-S93.

476

477 8. Stefanyshyn DJ, Nigg GM. lnfluence of midsole bending stiffness on joint energy and jump 478 height performance. Med Sci Sports Exerc. 2000;32(2):471-6.

479

480 9. Stefanyshyn D, Fusco C. Increased shoe bending stiffness increases sprint performance. 481 Sports Biomech. 2004;3(1):55-66.

482

483 10. Tinoco N, Bourgit D, Morin JB. Influence of midsole metatarsophalangeal stiffness on 484 jumping and cutting movement abilities. Proc Inst Mech Eng P J Sports Eng Technol. $485 \quad 2010 ; 224: 209-17$.

486

487 11. Boyer KA, Federolf P, Lin C, Nigg BM, Andriacchi TP. Kinematic adaptations to a variable 488 stiffness shoe: Mechanisms for reducing joint loading. J Biomech. 2012;45(9):1619-24. 489 
12. Burns AC, Kram R. The effect of cycling shoes and the shoe-pedal interface on maximal

491 mechanical power output during outdoor sprints. Footwear Sci. 2020;12:185-92.

492

493 13. Cruz CF, Bankoff AD. Electromyography in cycling: difference between clipless pedal 494 and toe clip pedal. Electromyography Clin Neurophysiol. 2001;41(4):247-52.

495

496 14. Mornieux G, Stapelfeldt B, Gollhofer A, Belli A. Effects of pedal type and pull-up action 497 during cycling. Int J Sports Med. 2008;29:817-22.

498

499

15. Ostler LM, Betts JA, Gore CJ. Gross cycling efficiency is not altered with and without toe$500 \quad$ clips. J Sports Sci. 2008;26:47-55.

501

502

16. Straw AH, Kram R. Effects of shoe type and shoe-pedal interface on the metabolic cost of 503 bicycling. Footwear Sci. 2016;8(1):19-22.

504

505 17. Hurt III JW, Kram R. No effect of cycling shoe sole stiffness on sprint performance.

506 Footwear Sci. 2021;13:69-77.

507

508 18. Anderson JC, Sockler JM. Effects of orthoses on selected physiologic parameters in cycling. 509 J Am Podiatr Med Assoc. 1990;80(3):161-6.

510

511 19. Fletcher JR, Asmussen MJ, Nigg SR, MacIntosh BR, Nigg BM. The effect of torsional shoe 512 sole stiffness on knee moment and gross efficiency in cycling. J sports Sci. 2019;37(13):145751363. 
20. Koch M, Frohlich M, Emrich E, Urhausen A. The impact of carbon insoles in cycling on

516 performance in the Wingate anaerobic test. J Sci Cycling. 2013;2(2):2-5.

517

518 21. Hurt III JW. Cycling shoe sole stiffness: effects on sprint performance and preliminary 519 analysis of strain distribution. Thesis of the University of Colorado, USA. 2015.

22. Dickson TB. Preventing overuse cycling injuries. Phys Sportsmed. 1985;13(10):116-23.

23. Mellion MB. Common Cycling Injuries, Management and Prevention. Sports Med. 1991;11(1):52-70.

24. Milani TL, Hennig EM, Lafortune MA. Perceptual and biomechanical variables for running in identical shoe constructions with varying midsole hardness. Clin Biomech. 1997;12(5):294528300.

25. Powell B. Medical aspects of racing. In: Burke ER, editor. Science of cycling. Human Kinetics. Champaign IL; 1986. P.185-201.

532

26. Quesada PM, Rash GS. Quantitative assessment of simultaneous capacitive and resistive 534 plantar pressure measurements during walking. Foot Ankle Int. 2000;21(11):928-34.

536 27. Sanderson DJ, Black AH, Montgomery J. The effect of varus and valgus wedges on coronal 537 plane knee motion during steady state cycling. Clin J Sport Med. 1994;4(2):120-4. 
28. Sanderson DJ, Hennig EM, Black AH. The influence of cadence and power out- put on

540 force application and in-shoe pressure distribution during cycling by competitive and 541 recreational cyclists. J Sports Sci. 2000;18(3):173-81.

29. Munera M, Chiementin X, Crequy S, Bertucci, W. Physical risk associated with vibration 544 at cycling. Mechanics Indust. 2014;15(6):535-40.

30. Uden H, Jones S, Grimmer K. Foot Pain and Cycling: a survey of frequency, type, location, associations and amelioration of foot pain. J Sci Cycling. 2012;1(2):28-34.

31. Vanwalleghem J, De Baere I, Loccufier M, Van Paepegem W. Sensor design for outdoor 550 racing bicycle field testing for human vibration comfort evaluation. Meas Sci Technol. 2013;24(9):095002.

32. Jaafar H, Attiogbé E, Rouis M, Vandewalle H, Driss T. Reliability of force-velocity tests in 554 cycling and cranking exercises in men and women. BioMed Research Int. 2015; 555 http://dx.doi.org/10.1155/2015/954780

33. Hausswirth C. Variabilités des profils de puissance en cyclisme et leur influence en course 558 à pied chez des triathlètes en préparation olympique. INSEP Report 8, 2011.

560 34. Hintzy F, Belli A, Grappe F, Rouillon JD. Effet de l'utilisation de pédales automatiques sur 561 les caractéristiques mécaniques mesurées lors de sprints sur cycloergomètre non isocinétique. 562 Science \& Sports 1999;14(3):137-44. 
564 35. Wewers ME, Lowe NK. A critical review of visual analogue scales in the measurement of 565 clinical phenomena. Res Nurs Health. 1990;13(4):227-36.

567 36. Cohen J. The effect size. Statistical power analysis for the behavioral sciences. In: Lawrence 568 Erlbaum Associates Publishers. 1988. p.77-83.

569

570 37. Arsac LM, Belli A, Lacour JR. Muscle function during brief maximal exercise: accurate 571 measurements on a friction-loaded cycle ergometer. Eur J Appl Physiol Occup Physiol. $572 \quad 1996 ; 74(1-2): 100-6$.

573

574 38. Hamley EY, Thomas V. Physiological and postural factors in the calibration of the bicycle 575 ergometer. J Physiol. 1967;191(2):55-6.

576

577 39. Vandewalle H, Peres G, Heller J, Panel J, Monod H. Force-velocity relationship and 578 maximal power on a cycle ergometer. Eur J Appl Physiol Occup Physiol. 1987;56(6):650-6. 579

580 40. Jorge M. Hull ML. Analysis of EMG measurements during bicycle pedaling. J Biomech. $581 \quad 1986 ; 19: 683-94$. 